\title{
Positive Mass Theorems for Black Holes
}

\author{
G. W. Gibbons ${ }^{1}$, S. W. Hawking ${ }^{1}$, Gary T. Horowitz ${ }^{2, \star}$, and Malcolm J. Perry ${ }^{3, \star \star}$ \\ 1 D.A.M.T.P., University of Cambridge, Silver Street, Cambridge CB39EW. England \\ 2 Institute for Advanced Study, Princeton, NJ 08540, USA \\ 3 Dept. of Physics, Princeton University, NJ 08544, USA
}

\begin{abstract}
We extend Witten's proof of the positive mass theorem at spacelike infinity to show that the mass is positive for initial data on an asymptotically flat spatial hypersurface $\Sigma$ which is regular outside an apparent horizon $H$. In addition, we prove that if a black hole has electromagnetic charge, then the mass is greater than the modulus of the charge. These results are also valid for the Bondi mass at null infinity. Finally, in the case of the Einstein equation with a negative cosmological constant, we show that a suitably defined mass is positive for data on an asymptotically anti-de Sitter surface $\Sigma$ which is regular outside an apparent horizon.
\end{abstract}

\section{Introduction}

The gravitational potential energy of any system is always negative because gravity is an attractive force. In Newtonian theory one can shrink any system to an arbitrarily small size and make the total energy indefinitely negative. However it appears that one cannot do this according to the general theory of relativity. As one considers smaller and smaller configurations for the system, the potential energy becomes more negative but the total energy, i.e. the rest mass plus potential energy plus kinetic energy seems to remain positive. At a certain critical size an outer future trapped surface appears $[1,2]$. This is a closed spacelike 2-surface which is in such a strong gravitational field that the outgoing future directed light rays or null geodesics orthogonal to it are converging, i.e. they are being dragged back by the gravitational field. The outer boundary of the region on a spacelike hypersurface which contains outer future trapped surfaces is called the future apparent horizon $[2,3]$. By the singularity theorems (see [2]) the system must collapse to produce a spacetime singularity provided that certain physically reasonable conditions hold. According to the unproved but very plausible cosmic

$\star$ Albert Einstein Fellow

$\star \star$ Supported by National Science Foundation Grant PHY 80-19754 
censorship hypothesis, this singularity is not visible to observers at a distance but is hidden in a black hole the boundary of which is a null surface called an event horizon. It is also generally assumed that any black hole has positive mass which, by the equivalence principle, means positive total energy. It was therefore conjectured that any physically reasonable system would have positive total energy or mass.

In recent years this conjecture has been proved by Schoen and Yau $[4,5]$. Their proof was based on the calculus of variations applied to surfaces of extremal area and volume. Although the basic idea of the proof was simple, the details became rather complicated when one allowed for the most general fall-off conditions at infinity. A completely different proof was given by Witten [6] based on a spinor field which satisfied a Dirac-like equation on a spacelike 3-surface. Unlike the Schoen and Yau proof, which applied whether or not there were apparent horizons, the Witten proof, in its simplest form, worked only when there were no horizons on the initial surface. Witten pointed out that his proof would also work if there was a horizon which separated our region of the spacelike surface from another asymptotically flat region. In the case of a stationary or static black hole, the no-hair theorems (see $[2,7]$ for reviews) essentially prove that the metric must be one of the Kerr-Newman solutions. These can be analytically continued to internal asymptotically flat regions. However in a general nonstationary solution containing an apparent horizon, there is no reason to believe that one can analytically continue the solutions to another asymptotically flat region.

The purpose of this paper is to show how Witten's arguments can be extended to the case where black holes are present without assuming anything about what happens inside a black hole. We shall show that the total mass is positive for initial data on a spacelike surface $\Sigma$ which is regular outside a future or past apparent horizon $H$ provided that the dominant energy condition $[8,2]$ holds. We also show that the mass is positive in the case of a maximal initial surface $\Sigma$ which is regular outside some minimal 2-surface $H$, as was first proved by Schoen and Yau [4].

In addition, we prove that the square of the total mass of a spacetime containing a black hole is greater than or equal to the sum of the squares of the total electric and magnetic charges (in suitable units) provided that the matter obeys the dominant energy condition and the local charge density is not greater than the local energy density [9]. Similar proofs could also be given for the positivity of the Bondi Mass which is measured at null infinity instead of at spacelike infinity. To do this, one simply modifies the proofs given here according to the methods used in [10]. In the case of the Einstein equation with negative cosmological term, we prove that a black hole solution which tends asymptotically to anti-de Sitter space has a positive value of a quantity which can be interpreted as the mass in this situation [11]. The proofs will be given in terms of 4 component spinors which can be generalized immediately to black holes in higher dimensional spacetimes which admit spinors. In the appendix we give the key steps of the proofs in terms of 2 component spinors for the case of 4-dimensions. 


\section{Witten's Formula}

We shall use a metric $g_{\mu \nu}$ with signature $(-+++)$ and $\gamma$-matrices which obey

$$
\gamma^{\mu} \gamma^{v}+\gamma^{v} \gamma^{\mu}=2 g^{\mu v}
$$

Greek indices will run from 0 to 3 and Latin indices from 1 to 3 . Indices with hats will refer to an orthonormal frame, and those without will be coordinate indices. The $\gamma^{\hat{a}}$ matrices are hermitian and $\gamma^{\hat{0}}$ is anti-hermitian.

Consider a spacelike 3-surface $\Sigma$ with induced metric $h_{i j}$, embedded in the four dimensional spacetime $\mathscr{M}, g_{\mu v}$. We choose our tetrad so that the 0 -vector is orthogonal to $\Sigma$. One then has the following relation between the projection into $\Sigma$ of the 4-dimensional spinor covariant derivative, ${ }^{(4)} \nabla_{a}$, and the intrinsic 3-dimensional covariant derivative ${ }^{(3)} \nabla_{a}$ :

$$
{ }^{(4)} \nabla_{\hat{a}} \varepsilon=\left({ }^{(3)} \nabla_{\hat{a}}+\frac{1}{2} K_{\hat{a} \hat{b}} \gamma^{\hat{b}} \gamma^{\hat{0}}\right) \varepsilon,
$$

where $K_{\hat{a} \hat{b}}$ are the triad components of the second fundamental form of $\Sigma$. If one multiplies by $\gamma^{\hat{a}}$ and sets the result equal to zero, one obtains the Witten equation which can be rewritten as

$$
\gamma^{\hat{a}(3)} \nabla_{\hat{a}^{\varepsilon}}=-\frac{1}{2} K \gamma^{\hat{0}} \varepsilon
$$

where $K=K_{\hat{a}}^{\hat{a}}$. If one multiplies by $\varepsilon^{\dagger}$ († indicates hermitian conjugate), acts on the result with $\gamma^{\hat{b}(3)} \nabla_{\hat{b}}$, and uses the Ricci identity, one obtains

${ }^{(3)} \nabla_{a}\left(\varepsilon^{\dagger(4)} \nabla^{a} \varepsilon\right)=\left({ }^{(4)} \nabla_{a} \varepsilon\right)^{\dagger}\left({ }^{(4)} \nabla^{a} \varepsilon\right)+\frac{1}{4} \varepsilon^{\dagger}\left({ }^{(3)} R+K^{2}-K_{a b} K^{a b}+2^{(3)} \nabla_{a}\left(K^{a b}-h^{a b} K\right) \gamma_{b} \gamma^{\hat{0}}\right) \varepsilon,(4)$

where ${ }^{(3)} R$ is the Ricci scalar on $\Sigma$ of the induced metric $h_{i j}$.

The Einstein equation implies

$$
\begin{gathered}
{ }^{(3)} R+K^{2}-K_{a b} K^{a b}=16 \pi T_{\hat{0}} \hat{o}, \\
{ }^{(3)} \nabla_{a}\left(K^{a b}-h^{a b} K\right)=8 \pi T^{\hat{0} b} .
\end{gathered}
$$

The dominant energy condition $[2,8]$ states that

$$
T_{\hat{0} \hat{o}} \geqq\left|T_{\hat{\alpha} \hat{\beta}}\right| \text {. }
$$

With this condition the last term on the right hand side of Eq. (4) is non-negative. If one integrates Eq. (4) over the region of $\Sigma$ bounded by a 2 -surface $H$ and a 2-surface at infinity, one obtains

$$
\oint_{\infty} \varepsilon^{\dagger(4)} \nabla_{a} \varepsilon d A^{a}-\oint_{H} \varepsilon^{\dagger(4)} \nabla_{a} \varepsilon d A^{a} \geqq \int_{\Sigma}\left({ }^{(4)} \nabla_{a} \varepsilon\right)^{\dagger}\left({ }^{(4)} \nabla^{a} \varepsilon\right) d \Sigma .
$$

Witten's argument is then as follows. Let $\Sigma$ be asymptotically flat. Choose a spinor field $\varepsilon$ on $\Sigma$ which satisfies Eq. (3) and which approaches a covariantly constant non-zero spinor $\varepsilon_{0}$ at infinity. Then the first term on the left hand side of Eq. (8) is

$$
4 \pi \varepsilon_{0}^{\dagger}\left(P^{\hat{0}}+P_{\hat{a}} \gamma^{\hat{a}} \gamma^{\hat{0}}\right) \varepsilon_{0}
$$


where $P^{\hat{0}}$ and $P^{\hat{a}}$ are the ADM mass and momentum, respectively, of the spacetime $\mathscr{M}$.

If (9) is positive, it follows that

$$
P^{\hat{0}}>\left|P^{\hat{a}}\right| .
$$

This will be true in the case when there are no horizons on $\Sigma$. In the next section we shall show that if there is an apparent horizon on $\Sigma$, one can choose a boundary condition for $\varepsilon$ on $H$ such that there will exist solutions of the Witten equation which tend to a constant spinor $\varepsilon_{0}$ at infinity and which make the horizon term in Eq. (8) zero. This will show that the ADM mass of an asymptotically flat spacetime that contains black holes, must be positive.

If (9) is zero, then $P^{\alpha}$ is null. From (4)-(8), it then follows that on $\Sigma,{ }^{(4)} \nabla_{a} \varepsilon=0$, and the energy-momentum tensor is null (i.e., for some null vector $l^{\alpha}, T^{\alpha \beta}=l^{\alpha} l^{\beta}$ ). This implies that spacetime is flat. To show this, one can trivially modify the proof of [12] by the inclusion of the boundary condition discussed in Sect. 3.

\section{Boundary Conditions}

We consider first the problem of proving that there are solutions of the Witten equation (3) in the case where $\Sigma$ does not have an inner boundary. Rather then deal with spinor fields which tend asymptotically to a constant value at large distances on $\Sigma$, it is convenient to make a conformal transformation to compactify $\Sigma$ by adding a point, $I$, at infinity. Thus if $\Sigma$ were diffeomorphic to $R^{3}$ we would obtain a compact manifold $\tilde{\Sigma}$ diffeomorphic to $S^{3}$. The metric $\tilde{h}_{i j}$ on $\tilde{\Sigma}$ is conformally related to the metric $h_{i j}$ on $\Sigma$

$$
\tilde{h}_{i j}=\Omega^{4} h_{i j},
$$

where $\Omega=0$ at $I$ and $\Omega>0$ on $\Sigma$. Strictly speaking the metric $\tilde{h}_{i j}$ and the conformal factor $\Omega$ will be smooth only in the case that the 4-momentum is orthogonal to $\Sigma$, i.e. $P^{a}=0$. However the case of non-zero $P^{a}$ has been considered by other authors [13]. We are primarily concerned with the behavior on the horizon rather than at infinity, so we shall consider only the case that $P^{a}=0$.

The field $\tilde{\varepsilon}=\varepsilon / \Omega^{2}$ obeys the equation

$$
\left(\tilde{\gamma}^{\hat{a}(3)} \tilde{V}_{\hat{a}}+\frac{1}{2} \Omega^{-2} K \gamma^{\hat{0}}\right) \tilde{\varepsilon}=0,
$$

where $\tilde{\gamma}^{a}=\Omega^{-2} \gamma^{a}$ and ${ }^{(3)} \tilde{\nabla}_{\hat{a}}$ is the covariant derivative of the metric $\tilde{h}_{i j}$. The conformal factor required to compactify an asymptotically flat initial surface $\Sigma$ is of the form

$$
\Omega=\frac{1}{r}+O\left(\frac{1}{r^{2}}\right)
$$

where $r$ is an asymptotic radial coordinate. Thus provided that

$$
K=O\left(\frac{1}{r^{2}}\right)
$$


which is a reasonable assumption, the matrix $\Omega^{-2} K \gamma^{\hat{0}}$ will be bounded on $\tilde{\Sigma}$. The spinor field $\varepsilon$ obeys a skew adjoint elliptic equation (12) on $\tilde{\Sigma}$. Suppose that there were a solution, $\tilde{\varepsilon}_{1}$, of $(12)$ that was everywhere regular on $\tilde{\Sigma}$. Then $\Omega^{2} \tilde{\varepsilon}_{1}$ would be a solution of Witten's equation (3) on $\Sigma$ which approached infinity like $\frac{1}{r^{2}}$. This would lead to a contradiction with Eq. (8) because the surface terms on the left would be zero but the volume integral of $\left({ }^{(4)} \nabla_{a} \varepsilon\right)^{\dagger}\left({ }^{(4)} \nabla^{a} \varepsilon\right)$ would be positive. It therefore follows that the differential operator in Eq. (12) has a unique inverse or Green's function $G(x, I)$ on $\tilde{\Sigma}$. Given a spinor $\tilde{\varepsilon}_{0}$ at $I, \Omega^{2}(x) G(x, I) \tilde{\varepsilon}_{0}$ defines a spinor field on $\Sigma$ which obeys Witten's equation (3) and which approaches a constant spinor $\tilde{\varepsilon}_{0}$ at infinity.

We shall now consider the case where $\Sigma$ has an inner boundary $H$. We now wish to find a solution of Witten's equation (3) on the region $\Sigma$ outside $H$ with the same behavior at infinity and with some suitable boundary condition on $H$. By the previous compactification technique we can transform it into a problem of finding a Green's function for Eq. (12) on a compact manifold $\tilde{\Sigma}$ with a boundary $H$ (which may have more than one connected component if there is more than one black hole).

It is convenient to choose our tetrad so that the zero-vector is orthogonal to $\Sigma$, the one-vector is orthogonal to $H$ and pointing into $\Sigma$ and the 2 and 3 vectors lie in $H$. One can then multiply Eq. (3) by $\gamma^{\hat{1}}$ to obtain

$$
\begin{aligned}
& { }^{(3)} \nabla_{\hat{1}} \varepsilon=-\gamma^{\hat{1}} \gamma^{\hat{A}(3)} \nabla_{\hat{A}} \varepsilon-\frac{1}{2} K \gamma^{\hat{1}} \gamma^{\hat{0}} \varepsilon \\
& =-\gamma^{\hat{1}} \gamma^{\hat{A}(2)} \nabla_{\hat{A}} \varepsilon-\frac{1}{2}\left(J+K \gamma^{\hat{1}} \gamma^{\hat{0}}\right) \varepsilon,
\end{aligned}
$$

where capital Latin indices run over 2 and $3,{ }^{(2)} \nabla_{A}$ denotes the covariant derivative on spinors in $H$ with respect to the intrinsic metric and $J$ is the trace of the second fundamental form of $H$ in $\Sigma$. One can see from Eq. (16) that the derivative of $\varepsilon$ normal to $H$ is determined by the values of $\varepsilon$ on $H$. Thus if one imposed the boundary condition that $\varepsilon$ vanished on $H$, then it would have to vanish everywhere.

What one needs is a boundary condition which restricts the freedom of $\varepsilon$ on $H$ by half. The condition that we shall use is

$$
\gamma^{\hat{1}} \gamma^{\hat{0}} \varepsilon=\varepsilon \text {. }
$$

The eigenvalues of the Hermitian matrix $\gamma^{\hat{1}} \gamma^{\hat{0}}$ are \pm 1 so condition (17) does indeed restrict $\varepsilon$ by half on $H$. With this boundary condition the conformal Witten equation (12) on $\tilde{\Sigma}$ is skew-adjoint. Equation (12) together with the boundary condition (17) forms an elliptic boundary value problem [14].

The boundary term on $H$ in Eq. (8) can be expressed as

$$
-\frac{1}{8 \pi} \oint_{H} \varepsilon^{\dagger}\left(J+\left(K-K_{\hat{1} \hat{1}}\right) \gamma^{\hat{1}} \gamma^{\hat{0}}+2 \gamma^{\hat{1}} \gamma^{\hat{A}} \bigoplus_{\hat{A}}\right) \varepsilon d A
$$

where

$$
\bigoplus_{\hat{A}^{\varepsilon}}={ }^{(2)} \nabla_{\hat{A}^{\varepsilon}}+\frac{1}{2} K_{\hat{A} \hat{1}} \gamma^{\hat{1}} \gamma^{\hat{O}} \varepsilon .
$$


The operator $\bigoplus_{A}$ is the 4-component version of the "edth" operator, $\partial$, of Geroch et al. [15]. The operator $\gamma^{\hat{1}} \gamma^{\hat{A}} D_{\hat{A}}$ anticommutes with $\gamma^{\hat{1}} \gamma^{\hat{0}}$. Thus the last term in (18) is zero if (17) holds. The condition that $H$ be a future apparent horizon implies that $J+K-K_{\hat{1} \hat{1}}=0$. Thus the boundary term on $H$ in Eq. (8) vanishes if (17) holds.

We now discuss the existence of solutions to Witten's equation with this boundary condition. Suppose that Eq. (12) had a non-zero solution $\tilde{\varepsilon}_{1}$ with the boundary condition (17). Then $\Omega^{2} \tilde{\varepsilon}_{1}$ would be a spinor field on $\Sigma$ which obeyed the Witten equation (3), the boundary condition (17) on $H$, and which decreased to zero at infinity like $\frac{1}{r^{2}}$. This would again lead to a contradiction because the boundary terms would be zero. This shows that the conformal Witten equation (12) with the boundary condition (17) has no zero modes on $\tilde{\Sigma}$. This suggests that there exists a Green's function to this elliptic boundary value problem (see Sect. 10.6 of [14]). The existence of such a Green's function probably can be rigourously proved by the methods of Parker and Taubes [13]. Thus we obtain solutions of the Witten equation on $\Sigma$ which obey the boundary condition (17) and which approach a constant spinor at infinity. The boundary term on $H$ for these solutions will be zero. Thus the mass will be positive. If $H$ were a past apparent horizon one could use a similar boundary condition to (17) but with a minus sign.

The positivity of mass can be proved under slightly different conditions. Suppose that $\Sigma$ is a 3 -surface which is maximal, i.e. $K=0$. Then one can derive a slightly different identity:

$$
P^{\hat{0}} \varepsilon_{0}^{\dagger} \varepsilon_{0} \geqq \int_{\Sigma} \varepsilon^{\dagger}\left(T_{\hat{0}} \hat{o}+\frac{1}{16 \pi} K_{\hat{a} \hat{b}} K^{\hat{a} \hat{b}}\right) \varepsilon d \Sigma-\frac{1}{8 \pi} \oint_{H} \varepsilon^{\dagger}\left(J+2 \gamma^{\hat{1}} \gamma^{\hat{A}(2)} \nabla_{\hat{A}}\right) \varepsilon d A,
$$

$\gamma^{\hat{1}} \gamma^{\hat{0}}$ anticommutes with $\gamma^{\hat{1}} \gamma^{\hat{A}(2)} \nabla_{\hat{A}}$. Thus the surface term on the right hand side of (20) is zero if $J=0$, i.e. if $H$ is a minimal 2-surface in the maximal 3-surface. At each point of $H$ the future or past directed outgoing null geodesics orthogonal to $H$ will be nondiverging, depending on the sign of $K_{\hat{1}}$. However $H$ need not be either future or past trapped because the future directed null geodesics may be converging only over part of $H$ and the past directed null geodesics converging over the other part. Nevertheless the fact that one can prove that the mass is positive in this situation regardless of what lies inside $H$ suggests that $H$ must lie behind the future or past event horizon although no proof of this is known to us.

We have proved the positivity of the mass for black holes in the general theory of relativity in four dimensions. However the proof generalizes to black holes in spacetimes with any number of dimensions and signature $(-++\ldots+)$ provided that the corresponding Einstein equation and the dominant energy condition hold, and that the spacetime admits a spin structure. This may be relevant for KaluzaKlein theories.

\section{The Electromagnetic Case}

In this section we show if $\mathscr{M}$ contains a charged black hole hole, then $M^{2} \geqq Q^{2}+P^{2}$ where $Q$ is the electric charge and $P$ is the magnetic charge in Gaussian units with $G=c=1$ and $M$ is the norm of the ADM 4-momentum $P^{\mu}$. The proof is along 
similar lines to that given in the last two sections except that the covariant derivative acting on spinors is replaced by the super-covariant derivative

$$
{ }^{(4)} \hat{\nabla}_{\mu} \varepsilon={ }^{(4)} \nabla_{\mu} \varepsilon+\frac{i}{4} F_{\alpha \beta} \gamma^{\alpha} \gamma^{\beta} \gamma_{\mu} \varepsilon
$$

The Witten equation now takes the form

$$
\gamma^{\hat{a}(3)} \nabla_{\hat{a}} \varepsilon+\frac{1}{2} K \gamma^{\hat{0}} \varepsilon-\frac{i}{2} \gamma^{\hat{a}}\left(E_{\hat{b}}-\gamma_{\hat{5}} B_{\hat{b}}\right) \gamma^{\hat{0}} \gamma^{\hat{b}} \gamma_{\hat{a}} \varepsilon=0
$$

where

$$
\begin{gathered}
F_{\hat{b} \hat{0}}=E_{\hat{b}}, \\
F_{\hat{a} \hat{b}}=\varepsilon_{\hat{a} \hat{b} \hat{c}} B^{c}, \\
\gamma^{\hat{5}}=\gamma^{\hat{0}} \gamma^{\hat{1}} \gamma^{\hat{2}} \gamma^{\hat{3}} .
\end{gathered}
$$

Using the Maxwell and Einstein equations one then obtains the identity [9]

$$
\begin{aligned}
\oint_{\infty} \varepsilon^{\dagger}\left({ }^{(3)} \hat{\nabla}_{i} \varepsilon\right) d A^{i}= & \oint_{H} \varepsilon^{\dagger(4)} \hat{\nabla}_{i} \varepsilon d A^{i}+\int_{\Sigma}\left({ }^{(4)} \hat{V}_{a} \varepsilon\right)^{\dagger}\left({ }^{(4)} \hat{V}^{a} \varepsilon\right) d \Sigma \\
& +4 \pi \int_{\Sigma} \varepsilon^{\dagger}\left(T_{0 \hat{0}}^{(m)}+\gamma^{\hat{j}} \gamma^{\hat{0}} T_{\hat{0} \hat{j}}^{(m)}-i \gamma^{\hat{0}}\left(J_{\hat{0}}^{E}-\gamma^{5} J_{0}^{M}\right)\right) \varepsilon d \Sigma,
\end{aligned}
$$

where $T_{\mu \nu}^{(m)}$ is the energy momentum tensor of the matter fields, i.e. the total energy momentum tensor $T_{\mu \nu}$ minus the energy momentum tensor of the Maxwell field, $J_{\mu}^{E}$ is the electric current and $J_{\mu}^{M}$ the magnetic current. We shall assume that the matter energy-momentum tensor obeys the condition

$$
T_{\hat{o} \hat{o}}^{(m)} \geqq\left[T_{\hat{O} a}^{(m)} T_{\hat{0}}^{(m) a}+\left(J_{\hat{0}}^{E}\right)^{2}+\left(J_{\hat{0}}^{M}\right)^{2}\right]^{1 / 2} .
$$

This is just the local version of the inequality that we are going to prove between the total mass $M$, the electric charge $Q$, and the magnetic charge $P$. In ordinary Maxwell theory the local magnetic current $J_{\mu}^{M}$ will be zero but there may be a global magnetic charge because of the non-trivial topology of a black hole.

The surface term on $H$ in Eq. (26) can be expressed as

$$
-\frac{1}{8 \pi} \oint_{H} \varepsilon^{\dagger}\left(J+\left(K-K_{\hat{1} \hat{1}}\right) \gamma^{\hat{1}} \gamma^{\hat{0}}+2 \gamma^{\hat{1}} \gamma^{\hat{A}} \bigoplus_{\hat{A}}+2 i \gamma^{\hat{0}}\left(E_{\hat{1}}-\gamma^{\hat{5}} B_{\hat{1}}\right)\right) \varepsilon d A
$$

The matrix $\gamma^{\hat{0}} \gamma^{\hat{1}}$ anticommutes with $\gamma^{\hat{1}} \gamma^{\hat{A}} D_{A}, \gamma^{\hat{0}}$, and $\gamma^{\hat{0}} \gamma^{\hat{5}}$ so that the last two terms in (28) are zero if the boundary condition (17) holds. Thus the whole surface term will be zero if $H$ is a future apparent horizon. Similarly the surface term will be zero if $H$ is a past apparent horizon and one uses a boundary condition like (17) but with a minus sign.

The argument is now similar to that given before. We assume that $P_{\hat{a}}=0$. One can compactify $\Sigma$ by adding a point at infinity to obtain a smooth manifold $\Sigma$ with boundary $H$ on which $\tilde{\varepsilon}=\varepsilon / \Omega^{2}$ obeys the equation

$$
\tilde{\gamma}^{a(3)} \tilde{\nabla} \tilde{a} \tilde{\varepsilon}=-\Omega^{2} \frac{K}{2} \gamma^{\hat{0}} \tilde{\varepsilon}+\Omega^{2} \frac{i}{2}\left(\gamma^{\hat{0}}\left(E_{b}-\gamma^{\hat{5}} B b\right) \gamma^{a} \gamma^{b} \gamma_{a} \tilde{\varepsilon}\right)
$$


The coefficients on the right hand side of (29) will be bounded on $\tilde{\Sigma}$ and the equation will be elliptic with the boundary condition (17). As before one can show there are no zero modes for the equation or its adjoint. Thus one can use the Green's function of the infinity point to construct solutions of Eq. (22) on $\Sigma$ which obey the boundary condition (17) on $H$ and which approach a constant spinor $\varepsilon_{0}$ at infinity.

The surface term at infinity in Eq. (26) can be expressed as

$$
4 \pi \varepsilon_{0}^{\dagger}\left(M-i \gamma^{\hat{0}}\left(Q-\gamma^{\hat{5}} P\right)\right) \varepsilon_{0} .
$$

This must be non-negative for any $\varepsilon_{0}$ which implies that

$$
M \geqq\left(Q^{2}+P^{2}\right)^{1 / 2} \text {. }
$$

\section{The Cosmological Case}

In this section we shall consider solutions of the Einstein equation with a cosmological constant

$$
R_{\mu \nu}-\frac{1}{2} g_{\mu \nu} R+\Lambda g_{\mu \nu}=8 \pi T_{\mu \nu} .
$$

We are mainly interested in spacetimes which contain black holes, however, our results apply equally well to spacetimes without black holes.

There are black hole solutions to (32) with positive $\Lambda$ [16]. However these are asymptotic to de Sitter space and so do not have a spacelike infinity at which to define a mass. There are also black hole solutions with negative $\Lambda$ which are asymptotic to anti-de Sitter space. The simplest of these is the Schwarzschild anti-de Sitter metric

$$
d s^{2}=-\left(1-\frac{2 M}{r}-\frac{\Lambda r^{2}}{3}\right) d t^{2}+\frac{d r^{2}}{\left(1-\frac{2 M}{r}-\frac{\Lambda r^{2}}{3}\right)}+r^{2}\left(d \theta^{2}+\sin ^{2} \theta d \varphi^{2}\right) .
$$

At this stage the quantity $M$ should be regarded simply as a parameter. However we shall introduce an expression for the mass of an asymptotically anti-de Sitter spacetime which is the analogue of the mass in asymptotically flat spacetimes and which will equal $M$ for the metric (33). This mass is not conserved because spacelike infinity in asymptotically anti-de Sitter spaces is timelike [2] and so gravitational radiation can come in or go out at spatial infinity. Hence it is analogous to the Bondi mass in asymptotically flat spacetimes.

We shall consider metrics which have a conformal structure similar to that of anti-de Sitter space outside some bounded region. This means that they have a timelike spatial infinity $I$ with topology $S^{2} \times \mathbb{R}^{1}$. The future event horizon will be defined to be the boundary of the points from which it is possible to escape to $I$ in the future direction. With the usual weak energy condition on $T_{\mu \nu}$, and assuming cosmic censorship, any future trapped surface must lie behind the event horizon. We shall prove that the expression that we shall give for the mass is positive for initial data on an asymptotic anti-de Sitter surface $\Sigma$ which is regular outside a future apparent horizon $H$. 
As in the electromagnetic case we introduce a supercovariant derivative, which, in this case, is

$$
{ }^{(4)} \hat{\nabla}_{\mu}={ }^{(4)} \nabla_{\mu}+\frac{\mu}{2} \gamma_{\mu} \text {, }
$$

where

$$
3 \mu^{2}=-\Lambda
$$

In anti-de Sitter space the connection given by (34) is flat. This means that there are four linearly independent supercovariantly constant spinor fields. These can be realized in the following way.

Consider the hyperboloid

$$
-\left(X^{0}\right)^{2}+\left(X^{1}\right)^{2}+\left(X^{2}\right)^{2}+\left(X^{3}\right)^{2}-\left(X^{5}\right)^{2}=\frac{3}{\Lambda}
$$

in 5-dimensional flat space with signature -+++- . This has topology $S^{1} \times \mathbb{R}^{3}$ with the $S^{1}$ being timelike. Anti-de Sitter space is the universal covering space of the hyperboloid. It has topology $\mathbb{R}^{4}$ and no closed timelike lines. The 5-dimensional flat covariant derivative projected into the hyperboloid can be related to the 4-dimensional intrinsic covariant derivative by

$$
\begin{aligned}
{ }^{(5)} \nabla_{\mu} \varepsilon & ={ }^{(4)} \nabla_{\mu} \varepsilon+\frac{1}{2} K_{\mu \nu} \gamma^{v} \gamma^{\hat{5}} \varepsilon \\
& ={ }^{(4)} \nabla_{\mu} \varepsilon+\frac{1}{2} \mu \gamma_{\mu} \gamma^{5} \varepsilon,
\end{aligned}
$$

where $K_{\mu \nu}$ is the second fundamental form of the hyperboloid and the pentad (5-frame) is chosen so that the 5-direction is orthogonal to the hyperboloid and the $(0-3)$ directions lie in the hyperboloid. A constant spinor $\varepsilon_{0}$ in the 5-dimensional space will obviously satisfy

$$
{ }^{(5)} \nabla_{\mu} \varepsilon_{0}=0
$$

This $\varepsilon_{0}$ is readily related to a supercovariantly constant spinor $\varepsilon_{1}$ which satisfies

$$
\text { (4) } \hat{\nabla}_{\mu} \varepsilon_{1}=0
$$

by

$$
\varepsilon_{1}=\left(1+\gamma^{\hat{5}}\right) \varepsilon_{0}
$$

Thus there are four linearly independent supercovariantly constant spinors in anti-de Sitter space. [Note that $\left(\gamma^{\hat{5}}\right)^{2}=-1$, so that the determinant of $\left(1+\gamma^{\hat{5}}\right)$ is non-zero, i.e. $\left(1+\gamma^{\hat{5}}\right)$ is not a projection operator.]

In analogy with the electromagnetic case, we define the cosmological Witten equation to be

$$
\gamma^{a(4)} \hat{\nabla}_{a} \varepsilon=0
$$

In terms of the intrinsic 3-dimensional derivative, this is

$$
\gamma^{a(3)} \nabla_{a} \varepsilon+\frac{1}{2} K \gamma^{\hat{0}} \varepsilon+\frac{3}{2} \mu \varepsilon=0 \text {. }
$$


The Witten identity in this case is

$$
\begin{aligned}
{ }^{(3)} \nabla_{a}\left(\varepsilon^{\dagger(4)} \hat{V}^{a} \varepsilon\right)= & \left({ }^{(4)} \hat{\nabla}_{a} \varepsilon\right)^{\dagger}\left({ }^{(4)} \hat{\Gamma}^{a} \varepsilon\right) \\
& +\frac{1}{4} \varepsilon^{\dagger}\left({ }^{(3)} R+K^{2}-K_{a b} K^{a b}+6 \mu^{2}+2^{(3)} \nabla_{a}\left(K^{a b}-g^{a b} K\right) \gamma_{b} \gamma^{\hat{0}}\right) \varepsilon
\end{aligned}
$$

The constraint equations now are

$$
\begin{gathered}
{ }^{(3)} R+K^{2}-K_{a b} K^{a b}+6 \mu^{2}=16 \pi T_{\hat{0} \hat{0}}, \\
{ }^{(3)} \nabla_{a}\left(K^{a b}-g^{a b} K\right)=8 \pi T^{\hat{0} b} .
\end{gathered}
$$

Thus the last term on the right of (44) will be positive semi-definite provided that the energy momentum tensor obeys the dominant energy condition.

We now integrate (44) over a surface $\Sigma$ whose inner boundary is a future apparent horizon $H$ and which asymptotically resembles the $X^{0}=0$ surface in anti-de Sitter space. We thus obtain

$$
\oint_{\infty} \varepsilon^{\dagger(4)} \hat{\nabla}_{a} \varepsilon d A^{a} \geqq \oint_{H} \varepsilon^{\dagger(4)} \hat{\nabla}_{a} \varepsilon d A^{a} .
$$

One can show that if $\varepsilon$ satisfies the boundary condition (17), then the surface term on $H$ vanishes. The cosmological Witten equation (42) on $\Sigma$ asymptotically resembles the ordinary Witten equation (3) on an asymptotically null surface in an asymptotically flat spacetime. The existence of asymptotically constant solutions to equations of this type has recently been established [17].

Let $\varepsilon$ satisfy Eq. (42), boundary codition (17) and asymptotically approach a supercovariantly constant spinor $\varepsilon_{0}$. Since the horizon term in (47) vanishes, the surface integral at infinity will be positive semi-difinite and will be zero only if the space is exactly anti-de Sitter. We define $O(3,2)$ momenta $P^{5 \mu}$ and $P^{\alpha \beta}$ such that this surface term is [18]

$$
4 \pi \varepsilon_{0}^{\dagger}\left(P^{\hat{5} \mu} \gamma_{\mu} \gamma^{\hat{0}}+\gamma^{\hat{0}} P^{\alpha \beta} \gamma_{\alpha} \gamma_{\beta}\right) \varepsilon_{0} .
$$

In asymptotically Schwarzschild-anti-de Sitter spacetimes, $P^{5 i}=P^{\alpha \beta}=0$, and $P^{\hat{5} \hat{0}}$ can be interpreted as the total energy. The positivity of (48) then implies that

$$
P^{\hat{5} \hat{0}} \geqq 0 \text {. }
$$

We note that in Schwarzschild-anti-de Sitter, $P^{50}$ coincides with usual mass parameter $M$.

This definition of the mass $P^{\hat{s} \hat{0}}$ seems to agree with that of Abbott and Deser [11] who gave a similar argument for believing that the mass would be positive for asymptotically anti-de Sitter metrics without horizons.

\section{Appendix}

In this appendix, we outline the key steps of the positive mass theorems proved in this paper, in terms of two component spinors. Throughout the appendix, we follow the notation of Pirani [19]. It should be noted that this entails our use of the metric signature $(+---)$ which is opposite to that employed in the main body of this paper. 
We first consider uncharged black holes in asymptotically flat spacetimes. Let $\Sigma$ be an asymptotically flat slice which begins at an apparent horizon $H$. Let $t^{\mu}$ be the unit normal to $\Sigma$ and $D_{a}$ be the projection of the four-dimensional derivative into $\Sigma$. (This was previously denoted by ${ }^{(4)} \nabla_{a}$.) In this case one can prove the positivity of mass by considering only a single two component spinor $\alpha^{A}$. Witten's equation $[\mathrm{Eq} .(3)]$ is

$$
D_{A A^{\prime}} \alpha^{A}=0
$$

From this, it follows that

$$
-D_{m}\left(t^{A A^{\prime}} \bar{\alpha}_{A^{\prime}} D^{m} \alpha_{A}\right)=-t^{A A^{\prime}}\left(D_{m} \alpha_{A}\right)\left(D^{m} \bar{\alpha}_{A^{\prime}}\right)+4 \pi T_{\mu v} t^{\mu} k^{\nu}
$$

where $k^{v}=\alpha^{N} \bar{\alpha}^{N^{\prime}}$. If $T_{\mu \nu}$ obeys the dominant energy condition, then the right-hand side of (A2) is non-negative. Integration of (A2) over $\Sigma$ then yields

$$
\oint_{\infty}-t^{A A^{\prime}} \bar{\alpha}_{A^{\prime}} D_{m} \alpha_{A} d S^{m} \geqq \int_{H}-t^{A A^{\prime}} \bar{\alpha}_{A^{\prime}} D_{m} \alpha_{A} d S^{m} .
$$

If $\alpha^{A}$ asymptotically approaches a covariantly constant spinor $\alpha_{0}^{A}$, then the boundary term at infinity is $4 \pi P_{\mu} k_{0}^{\mu}$, where $P_{\mu}$ is the ADM four-momentum, and $k_{0}^{\mu}=\alpha_{0}^{M} \bar{\alpha}_{0}^{M^{\prime}}$.

To discuss the boundary condition for $\alpha^{A}$ on $H$, we introduce a spinor basis $\left(o^{A}, i^{A}\right)$ on $H$ such that $o^{A} \bar{o}^{A^{\prime}}$ and $i^{A} \bar{i}^{A^{\prime}}$ are the outgoing and ingoing null vectors orthogonal to $H, o^{A} \bar{i}^{A^{\prime}}$ is tangential to $H$, and $o_{A} i^{A}=1$. We now expand $\alpha^{A}$ in terms of this basis as

$$
\alpha^{A}=X o^{A}+Y i^{A}
$$

The boundary term on the horizon becomes [see Eq. (18)]

$$
\int_{H}[\varrho X \bar{X}+\mu Y \bar{Y}+2 \operatorname{Re} \bar{Y}(\delta X+\beta X) d A,
$$

where $\delta$ is the differential operator $\delta=o^{A} \bar{i}^{A^{\prime}} \nabla_{A A^{\prime}}, \varrho=o^{A} \bar{\delta} o_{A}$ is the convergence of outgoing null vectors, $\mu=i^{A} \delta i_{A}$ is the divergence of ingoing null vectors, and $\beta=i^{A} \delta o_{A}$. (Here $\delta+\beta$ is the Geroch-Held-Penrose [15] operator $\delta$, edth.) By definition, $\varrho=0$ on a future apparent horizon. The boundary condition that we impose on $H$ is [Eq. (17)]

$$
Y=\alpha^{A} o_{A}=0
$$

Thus, with this choice of boundary condition, the boundary term (A5) vanishes.

Therefore, if $\alpha^{A}$ is a solution to (A1) which satisfies (A6) on $H$, and approaches a constant spinor $\alpha_{0}^{A}$ asymptotically, then (A3) implies

$$
P_{\mu} k_{0}^{\mu} \geqq 0 .
$$

Since $k_{0}^{\mu}$ is an arbitrary null vector, this shows that $P_{\mu}$ is future directed. If $H$ was a past apparent horizon, then $\mu=0$ and one can use the boundary condition $X$ $=i^{A} \alpha_{A}=0$ on $H$ to establish a similar result. 
We next consider charged black holes in asymptotically flat spacetimes. Let $\Sigma$ and $D_{m}$ be as before. We define the Maxwell spinor $\varphi_{M N}$ by

$$
F_{\mu \nu}=\frac{1}{\sqrt{2}}\left(\varphi_{M N} \varepsilon_{M^{\prime} N^{\prime}}+\bar{\varphi}_{M^{\prime} N^{\prime}} \varepsilon_{M N}\right) .
$$

To treat this case, we need to introduce a pair of two component spinors $\left(\alpha^{A}, \beta_{A^{\prime}}\right)$. The supercovariant derivative defined by Eq. (21) is

$$
\begin{gathered}
\hat{\nabla}_{M M^{\prime}} \alpha_{A}=\nabla_{M M^{\prime}} \alpha_{A}+\varphi_{A M} \beta_{M^{\prime}}, \\
\hat{\nabla}_{M M^{\prime}} \beta_{A^{\prime}}=\nabla_{M M^{\prime}} \beta_{A^{\prime}}-\bar{\varphi}_{A^{\prime} M^{\prime}} \alpha_{M} .
\end{gathered}
$$

The Witten equation (22), is

$$
\begin{gathered}
\hat{D}_{A A^{\prime}} \alpha^{A}=D_{A A^{\prime}} \alpha^{A}-t_{A A^{\prime}} t^{B B^{\prime}} \varphi^{A}{ }_{B} \beta_{B^{\prime}}=0 \\
\hat{D}_{A A^{\prime}} \beta^{A^{\prime}}=D_{A A^{\prime}} \beta^{A^{\prime}}+t_{A A^{\prime}} t^{B B^{\prime}} \bar{\varphi}^{A^{\prime}{ }_{B^{\prime}}} \alpha_{B}=0 .
\end{gathered}
$$

If these equations are satisfied, then one obtains the identity (26):

$$
\begin{aligned}
-D_{m} & {\left[t^{A A^{\prime}} \bar{\alpha}_{A^{\prime}} \hat{D}^{m} \alpha_{A}+t^{A A^{\prime}} \bar{\beta}_{A} \hat{D}^{m} \beta_{A^{\prime}}\right] } \\
= & -t^{A A^{\prime}}\left[\left(\hat{D}_{m} \alpha_{A}\right)\left(\overline{\hat{D}^{m} \alpha_{A^{\prime}}}\right)+\left(\hat{D}_{m} \beta_{A^{\prime}}\right)\left(\hat{D}^{m} \beta_{A}\right)\right]+4 \pi T_{\mu \nu}^{(m)} t^{\mu} \xi^{v} \\
& +4 \pi\left[J_{\mu} t^{\mu}\left(\bar{\alpha}^{B^{\prime}} \beta_{B^{\prime}}\right)+\bar{J}_{\mu} t^{\mu}\left(\alpha^{B} \bar{\beta}_{B}\right)\right],
\end{aligned}
$$

where $T_{\mu \nu}^{(m)}$ is the matter energy-momentum tensor, $\xi^{\nu}=\alpha^{N} \bar{\alpha}^{N^{\prime}}+\beta^{N} \bar{\beta}^{N^{\prime}}$ and

$$
J_{A A^{\prime}}=\frac{1}{4 \pi} \nabla_{A^{\prime} B} \varphi^{B}{ }_{A}
$$

is a complex current which is related to the electric and magnetic currents $J_{\mu}^{E}$ and $J_{\mu}^{M}$ by

$$
J_{\mu}=\frac{1}{\sqrt{2}}\left(J_{\mu}^{E}-i J_{\mu}^{M}\right) .
$$

If $T_{\mu \nu}^{(m)}$ satisfies Eq. (27), then the right hand side of (A11) is non-negative. Integrating (A11) over $\Sigma$ yields

$$
\int_{\infty}-t^{A A^{\prime}}\left(\bar{\alpha}_{A^{\prime}} \hat{D}_{m} \alpha_{A}+\bar{\beta}_{A} \hat{D}_{m} \beta_{A^{\prime}}\right) d S^{m} \geqq \int_{H}-t^{A A^{\prime}}\left(\bar{\alpha}_{A^{\prime}} \hat{D}_{m} \alpha_{A}+\bar{\beta}_{A} \hat{D}_{m} \beta_{A^{\prime}}\right) d S^{m} .
$$

The boundary term is identical to the uncharged case [Eq. (A5) for $\alpha^{A}$ and $\beta_{A^{\prime}}$ ] apart from the addition of the term

$$
\int\left[\phi_{A B}\left(\bar{\alpha}^{M^{\prime}} \beta_{M^{\prime}}\right) \varepsilon_{A^{\prime} B^{\prime}}+\bar{\phi}_{A^{\prime} B^{\prime}}\left(\alpha^{M} \bar{\beta}_{M}\right) \varepsilon_{A B}\right] t^{b} d S^{a} .
$$

On $H$, if both $\alpha^{A}$ and $\beta_{A^{\prime}}$ satisfy the boundary condition (A6) then this extra term vanishes. So the entire boundary integral on $H$ again vanishes. At infinity, if $\alpha^{A}$ and $\beta_{A^{\prime}}$ approach the supercovariantly constant spinors $\alpha_{0}^{A}$ and $\beta_{0 A^{\prime}}$, then the boundary term becomes

$$
4 \pi\left[P_{\mu} \xi_{0}^{\mu}+\sqrt{2} Q \operatorname{Re}\left(\alpha_{0}^{A} \bar{\beta}_{0 A}\right)+\sqrt{2} P \operatorname{Im}\left(\alpha_{0}^{A} \bar{\beta}_{0 A}\right)\right] .
$$


Equation (A14) states that this must be positive for all $\alpha_{0}^{A}, \beta_{0 A^{\prime}}$. Hence

$$
P_{\mu} P^{\mu} \geqq Q^{2}+P^{2} \text {. }
$$

Finally, we consider black holes in asymptotically anti-de Sitter spacetimes. The supercovariant derivative in this case is [Eq. (34)]

$$
\begin{aligned}
& \hat{\nabla}_{M M^{\prime}} \alpha_{A}=\nabla_{M M^{\prime}} \alpha_{A}+K \varepsilon_{M A} \beta_{M^{\prime}}, \\
& \hat{\nabla}_{M M^{\prime}} \beta_{A^{\prime}}=\nabla_{M M^{\prime}} \beta_{A^{\prime}}+K \varepsilon_{M^{\prime} A^{\prime}} \alpha_{A},
\end{aligned}
$$

where $K^{2}=-\frac{1}{6} \Lambda$. (We define our cosmological constant $\Lambda$ such that it is negative for anti-de Sitter spacetime.) Let $\Sigma$ be a spacelike surface from $H$ which asymptotically resembles the $X^{0}=0$ surface in anti-de Sitter spacetime. The Witten equation in this case (43) is

$$
\begin{aligned}
& \hat{D}_{A A^{\prime}} \alpha^{A}=D_{A A^{\prime}} \alpha^{A}+\frac{3}{2} K \beta_{A^{\prime}}=0 \\
& \hat{D}_{A A^{\prime}} \beta^{A^{\prime}}=D_{A A^{\prime}} \beta^{A^{\prime}+\frac{3}{2}} K \alpha_{A}=0
\end{aligned}
$$

These equations imply (44) that

$$
-D_{m}\left[t^{A A^{\prime}}\left(\bar{\alpha}_{A^{\prime}} \hat{D}^{m} \alpha_{A}+\bar{\beta}_{A} \hat{D}^{m} \beta_{A^{\prime}}\right)\right]=-t^{A A^{\prime}}\left[\hat{D}_{m} \alpha_{A} \overline{\hat{D}^{m} \alpha_{A^{\prime}}}+\hat{D}_{m} \beta_{A^{\prime}} \overline{\hat{D}^{m} \beta_{A}}\right]+4 \pi T_{\mu v} t^{\mu} \xi^{\nu}
$$

If $T_{\mu \nu}$ satisfies the dominant energy condition, then the right-hand side is non-negative. We now integrate (A20) over $\Sigma$. If $\alpha^{A}, \beta_{A^{\prime}}$ satisfy boundary condition (A6) then the horizon terms vanishes. If they asymptotically approach supercovariantly constant spinors $\alpha_{0}^{A}$ and $\beta_{0 A^{\prime}}$, then the surface term at infinity is finite, and can be related to $P^{5 \mu}, P^{\alpha \beta}$ in the same way as Eq. (48).

Acknowledgements. We are grateful for discussions with a large number of colleagues. While this paper was in preparation we learnt of similar work by P. Tod and O. Reula.

\section{References}

1. Penrose, R.: Gravitational collapse and spacetime singularities. Phys. Rev. Lett. 14, 57-59 (1965)

2. Hawking, S.W., Ellis, G.F.R.: The large scale structure of space-time. Cambridge: Cambridge University Press 1973

3. Hawking, S.W.: The event horizon in: Black holes, pp. 1-55. de Witt, B.S., de Witt, C.M. (eds.) New York: Gordon and Breach 1973

4. Schoen, R., Yau, S.-T.: On the proof of the positive mass conjecture in general relativity. Commun. Math. Phys. 65, 45-76 (1979)

5. Schoen, R., Yau, S.-T.: Proof of the positive mass theorem. II. Commun. Math. Phys. 79, 231-260 (1981)

6. Witten, E. : A new proof of the positive energy theorem. Commun. Math. Phys. 80, 381-402 (1981)

7. Carter, B.: The general theory of black hole properties. (eds.). In: General relativity, pp. 294-369. Hawking, S.W., Israel, W., Cambridge: Cambridge University Press 1979

8. Hawking, S.W.: The conservation of matter in general relativity. Commun. Math. Phys. 18, 301$306(1970)$

9. Gibbons, G.W., Hull, C.M.: A Bogomolny bound for general relativity and solutions in $N=2$ supergravity. Phys. Lett. 109 B, 190-194 (1982)

10. Horowitz, G.T., Perry, M.J.: Gravitational energy cannot become negative. Phys. Rev. Lett. 48, 371-374 (1982) 
11. Abbott, G., Deser, S.: Stability of gravity with a cosmological constant. Nucl. Phys. B 195, 76-96 (1982)

12. Ashtekar, A., Horowitz, G.T.: Energy-momentum of isolated systems cannot be null. Phys. Lett. 89 A, 181-183 (1982)

13. Parker, T., Taubes, C.H.: On Witten's proof of the positive energy theorem. Commun. Math. Phys. 84, 223-238 (1982)

Reula, O.: Existence theorem for solutions of Witten's equations and nonnegativity of total mass. J. Math. Phys. 23, 810-814 (1982)

14. Hormander, L.: Linear partial differential operators. New York: Academic Press 1963

15. Geroch, R., Held, A., Penrose, R. : A space-time calculus based on pairs of null directions. J. Math. Phys. 14, 874-881 (1973)

16. Gibbons, G.W., Hawking, S.W.: Cosmological event horizons, thermodynamics, and particle creation. Phys. Rev. D 15, 2738-2751 (1977)

17. Reula, O., Tod, P.: Private communication

18. Freedman, D.Z.: private communication.

19. Pirani, F.A.E.: Introduction to gravitational radiation theory. In: Lectures on General Relativity, pp. 249-373. Trautman, A., Pirani, F.A.E., Bondi, H. (eds.). Englewood Cliffs, N.J.: Prentice-Hall 1965

Communicated by A. Jaffe

Received June 18, 1982; in revised form November 1, 1982 